\title{
Pretreatment neutrophil-to-lymphocyte ratio in peripheral blood was associated with pulmonary tuberculosis retreatment
}

\author{
Yongmei Yin ${ }^{1}$, Shougang Kuai ${ }^{2}$, Jun Liư ${ }^{2}$, YingYing Zhang ${ }^{2}$, Zhongbao Shan², Lan Gu', \\ Qiusheng Huang ${ }^{3}$, Hao Pei², Jun Wang ${ }^{2}$
}

\author{
${ }^{1}$ Radiology Department, The Fifth People's Hospital of Wuxi, Jiangnan University, \\ Wuxi, Jiangsu, China \\ ${ }^{2}$ Center of Clinical Laboratory, The Fifth People's Hospital of Wuxi, Jiangnan University, \\ Wuxi, Jiangsu, China \\ ${ }^{3}$ Department of Respiratory Medicine, The Fifth People's Hospital of Wuxi, Jiangnan \\ University, Wuxi, Jiangsu, China
}

Submitted: 1 July 2015

Accepted: 23 August 2015

Arch Med Sci 2017; 13, 2: 404-411

DOI: https://doi.org/10.5114/aoms.2016.60822

Copyright (๑) 2016 Termedia \& Banach

\section{Abstract}

Introduction: The neutrophil-to-lymphocyte ratio (NLR) is a readily available biomarker associated with recurrence and survival in various diseases. The objective of this study was to investigate the relationship between NLR and pulmonary tuberculosis (PTB) retreatment.

Material and methods: This was a case-control study that included 306 newly diagnosed cases of PTB in the clinical database of the Infectious Hospital of Wuxi from December 2009 to December 2011. Of the 306 patients, a total of 68 cases were followed up with TB retreatment. The remaining 238 PTB patients who completed anti-TB treatment and were cured without retreatment were selected as controls.

Results: According to the ROC curve, the best cut-off value of NLR was 2.53, with a sensitivity of $70.6 \%$ and a specificity of $45.4 \%$. The NLR $\geq 2.53$ before anti-TB treatment was associated with PTB retreatment $(\mathrm{OR}=1.994$, $95 \% \mathrm{Cl}: 1.116-3.564$; adjusted $\mathrm{OR}(\mathrm{AOR})=2.409,95 \% \mathrm{Cl}: 1.212-4.788)$. The retreatment rates with NLR $\geq 2.53$ and NLR $<2.53$ were $27.1 \%$ and $15.5 \%$, respectively, with a significant difference (log-rank test; $p=0.010$ ). Additionally, cavitation on chest X-ray $(\mathrm{OR}=2.922,95 \% \mathrm{Cl}: 1.654-5.411$; $\mathrm{AOR}=2.482,95 \% \mathrm{Cl}: 1.230-5.007)$, history of smoking $(\mathrm{OR}=2.202,95 \% \mathrm{Cl}$ : $1.158-3.493 ; \mathrm{AOR}=2.321,95 \% \mathrm{Cl}: 1.135-4.745)$ and age $\geq 60(\mathrm{OR}=3.828$, $95 \% \mathrm{Cl}: 1.626-9.015$; $\mathrm{AOR}=2.931,95 \% \mathrm{Cl}: 1.122-7.653)$ were also associated with PTB retreatment.

Conclusions: NLR $\geq 2.53$ is predictive of PTB retreatment. Otherwise, initial cavitation on chest $X$-ray, history of smoking, and age of $\geq 60$ are also risk factors for PTB retreatment.

Key words: neutrophil-to-lymphocyte ratio, pulmonary tuberculosis, retreatment.

\section{Introduction}

According to the WHO Global Tuberculosis Report 2014, an estimated 0.4 million people had already been diagnosed with tuberculosis (TB) but developed to be retreatment cases [1]. Tuberculosis is present in all

\author{
Corresponding authors: \\ Jun Wang \\ Hao Pei \\ Center of Clinical Laboratory \\ The Fifth People's \\ Hospital of Wuxi \\ Jiangnan University \\ 214005 Wuxi, Jiangsu, China \\ Phone: +86-0510-82019555- \\ 8201 \\ E-mail: j.wang1988@hotmail. \\ com, \\ peihao1008@126.com
}


regions of the world. India and China accounted for $22 \%$ and $15 \%$ of new and relapse cases, respectively [1]. Currently, more than $85 \%$ of initial patients are cured in China, with an increase in coverage of directly observed treatment short course (DOTS) therapy [2]. According to national tuberculosis prevalence surveys in China, the proportion treated by the public health system (using the DOTS strategy) increased from 15\% in 2000 to $66 \%$ in 2010 [2]. However, there still existed massive TB retreatment of patients in China; the proportion of retreatment cases was 31\% in 2010 [2], due to nosocomial transmission, the use of inadequate treatment regimens, poor management of resistant cases and the high migration rate. Moreover, with the large overall number of TB patients and the limited cure rate for TB retreatment, the high cost of retreatment should not be ignored in China.

Retreatment of TB is presenting new challenges for TB control in this context. The type of retreatment of TB included relapse, failure, treatment after loss to follow-up and other previously treated patients [1]. Relapse of TB is one of the most concerning issues [3]. The incidence of TB relapse in those who underwent previous treatment can be 30 times higher than the incidence of TB in new cases [4]. The most concerning situation is multidrug resistant (MDR) TB, the retreatment of patients who are often exposed to conditions associated with future failures that are attributable to microbial resistance. In the recent global surveillance for drug-resistant TB, it was estimated that $7.9 \%$ of relapse cases were MDR TB [5]. Retreatment patients also have a lower cure rate than new cases and experience more side effects during treatment with the second-line drugs [6].

Better understanding of the determinants of TB retreatment helps to identify those at high risk of disease retreatment and promises to reduce the disease burden through risk factor intervention. Recently, several studies have suggested that neutrophil-to-lymphocyte ratio (NLR), plateletto-lymphocyte ratio (PLR), C-reactive protein $(C R P)$, platelet count, plateletcrit (PCT), and platelet distribution width (PDW) may be potential biomarkers of PTB severity [7-10]. Among these, the neutrophil-to-lymphocyte ratio (NLR) is a readily available and cost-effective biomarker used in clinical practice that has been associated with recurrence [11, 12] and survival [13-15] in various diseases. However, whether NLR is a predictive factor associated with TB retreatment has not yet been ascertained. The objective of this study was to investigate the value of NLR to predict pulmonary tuberculosis (PTB) retreatment coordinated with other risk factors.

\section{Material and methods}

\section{Study population and ethical approval}

We conducted a case-control study of all newly diagnosed cases of PTB in the clinical database of The Fifth People's Hospital of Wuxi from December 2009 to December 2011. Following the national TB guidelines, a case of PTB was defined by positive sputum smear or sputum culture or, in the absence of bacteriological evidence, positive chest X-ray compatible with PTB plus clinical improvement after anti-TB treatment. Of the 386 incident cases of PTB during the study period, we excluded patients who transferred out $(n=32)$, died during anti-TB treatment $(n=20)$ and those for whom information was missing $(n=28)$ for any other reasons during the follow-up period. After exclusion, 306 patients with complete follow-up information until December $31^{\text {st }}$ of 2014 were included in this study. Meanwhile, 68 patients were followed up for TB retreatment (retreatment was defined as patients undergoing the second anti-TB treatment with active PTB after previous anti-TB treatment for one month or more). The rest of the no retreatment PTB cases $(n=238)$ who had completed anti-TB treatment and were cured without retreatment were selected as controls (Figure 1). Demographic and clinical data of the 306 PTB patients were obtained in the present study (Table I).

This study was approved by the Institutional Ethics Committee of The Fifth People's Hospital of Wuxi, Affiliated to Jiangnan University (No: QD2014-0023), and was in compliance with the national legislation and the Declaration of Helsinki

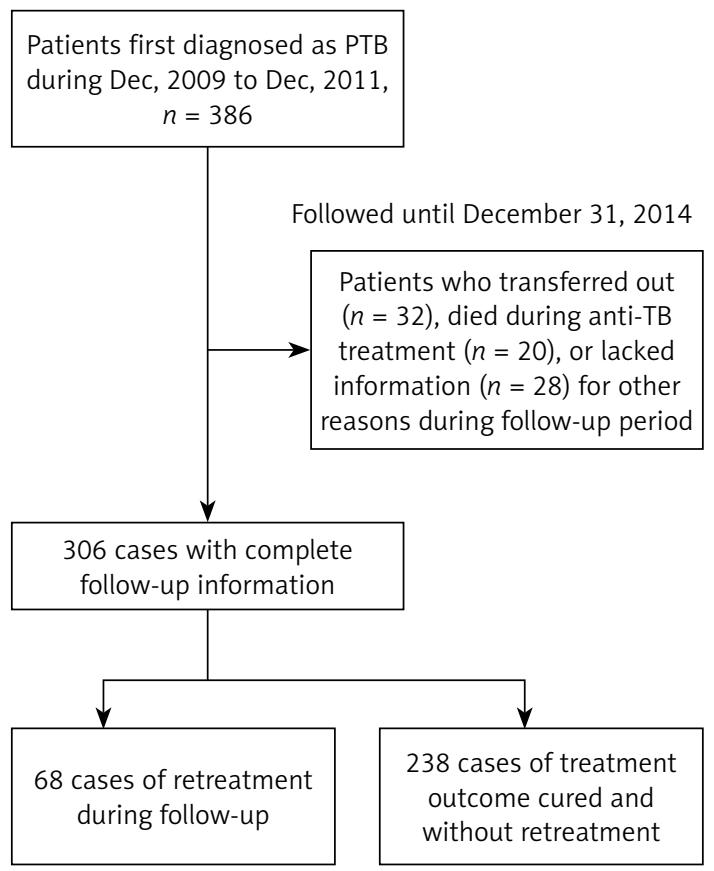

Figure 1. Flow diagram for enrollment of study participants in the nested case-control study 
Table I. Characteristics of study participants

\begin{tabular}{|c|c|}
\hline Parameter & Patients $(n=306)$ \\
\hline Age $\left[\right.$ years] ${ }^{\mathrm{a}}$ & $59(40-70)$ \\
\hline Sex, maleb & $228(74.5)$ \\
\hline WBC count $\left[\times 10^{9} / I\right]^{\mathrm{a}}$ & $6.935(5.483-8.907)$ \\
\hline Neutrophil count $\left[\times 10^{9} / 1\right]^{a}$ & $4.630(3.437-6.432)$ \\
\hline Lymphocyte count $\left[\times 10^{9} / 1\right]^{a}$ & $1.497(1.113-1.860)$ \\
\hline Monocyte count $\left[\times 10^{9} / 1\right]^{a}$ & $0.462(0.224-0.660)$ \\
\hline$N L R^{a}$ & $3.124(2.131-4.917)$ \\
\hline \multicolumn{2}{|l|}{ History of smokingb: } \\
\hline Yes & $150(49.0)$ \\
\hline No & $156(51.0)$ \\
\hline \multicolumn{2}{|l|}{ Cancer $^{\mathrm{b}}$ : } \\
\hline Yes & $14(4.6)$ \\
\hline No & $292(95.4)$ \\
\hline \multicolumn{2}{|l|}{$\mathrm{HIV}^{\mathrm{b}}:$} \\
\hline Yes & $5(1.6)$ \\
\hline No & $301(98.4)$ \\
\hline \multicolumn{2}{|l|}{$\mathrm{DM}^{\mathrm{b}}:$} \\
\hline Yes & $32(10.5)$ \\
\hline No & $274(89.5)$ \\
\hline \multicolumn{2}{|l|}{$\mathrm{HBV} / \mathrm{HCV}^{\mathrm{b}}$ : } \\
\hline Yes & $37(12.1)$ \\
\hline No & $269(87.9)$ \\
\hline \multicolumn{2}{|l|}{$\begin{array}{l}\text { Coexisting extra-pulmonary } \\
\text { lesion }{ }^{\text {b: }}\end{array}$} \\
\hline Yes & $62(20.3)$ \\
\hline No & $244(79.7)$ \\
\hline \multicolumn{2}{|l|}{$\begin{array}{l}\text { Culture positive and/or smear- } \\
\text { positiveb,\#: }\end{array}$} \\
\hline Yes & $127(41.5)$ \\
\hline No & $160(52.3)$ \\
\hline \multicolumn{2}{|l|}{ Cavitation on initial $C X R^{b, \&}$ : } \\
\hline Yes & $97(31.7)$ \\
\hline No & $194(63.4)$ \\
\hline
\end{tabular}

${ }^{a}$ Data are presented as numbers (\%) of individuals except where indicated. ${ }^{b}$ Data are presented as median value (interquartile range). "Missing information in 19 patients. ${ }^{8}$ Missing information in 15 patients.

guidelines. Because it was a retrospective review of the TB registry and medical records, informed consent was not available. All patients' records and information were anonymized and de-identified prior to analysis.

\section{Measurement of NLR and other factors}

The NLR was directly calculated from peripheral blood counts. Peripheral blood collected in eth- ylene-diamine tetra-acetic acid (EDTA)-containing tubes was analyzed by one of two clinical diagnostic laboratories each using a five-part differential hematology analyzer (Sysmex Model XS, Hamburg, Germany). Full blood count measurement was subject to strict quality assurance procedures including twice-daily high and low internal quality control, fortnightly quality controls through the Wuxi clinical laboratory QC scheme (Wuxi, Jiangsu, China) and annual quality assurance as part of the Jiangsu clinical laboratory QC scheme. Both laboratories are accredited by the China National Accreditation System in accordance with international standards ISO 17025/2005 and ISO 15189/2007. Information on other factors was also obtained from the clinical database of the Infectious Hospital of Wuxi. We extracted the following information through medical chart review: age, sex, history of smoking, HIV status, cancer, diabetes mellitus (DM), HBV/HCV status, coexisting extra-pulmonary TB, bacteriological confirmation and cavitation on chest X-ray within 1 month of initiating anti-TB treatment.

\section{Statistical analysis}

Statistical analysis was conducted using SPSS version 17.0. Pearson's $\chi^{2}$ test was conducted to identify significant differences across PTB retreatment cases and controls. The odds ratios (ORs) were estimated with 95\% confidence intervals (Cls) from the bivariate analysis to evaluate the factors associated with PTB retreatment cases and controls. Adjusted odds ratios (AOR) with $95 \% \mathrm{Cls}$ were estimated from the multivariate analysis to determine the factors associated with PTB retreatment. The cumulative recurrence rate during the follow-up period was calculated using Kaplan-Meier analysis, and the log-rank test was used to examine the significance of the differences between different NLR groups. $P$-value $<0.05$ was considered as statistically significant for all analyses.

\section{Results}

The baseline characteristics of the patient population included in this study are outlined in Table I. There were 306 patients enrolled in the study, with a median age of 59 years. Of the 306 patients, the majority of patients were male (74.5\%). There were 150 (49\%) patients with a history of smoking, 37 (12.1\%) HBV/HCV-positive patients, 32 (10.5\%) patients diagnosed with diabetes mellitus (DM), 14 (4.5\%) patients with cancer and only 5 (1.6\%) patients with HIV. There were 62 PTB patients with coexisting extra-pulmonary lesions, 127 patients bacteriologically confirmed positively and 97 patients diagnosed with cavitation on initial chest X-ray (CXR). Full blood counts were obtained for all included patients. The median 
WBC for the study population was $6.935 \times 10^{9} / 1$ (interquartile range (IQR): $5.483-8.907 \times 10^{9} / \mathrm{l}$ ), the median neutrophil count was $4.630 \times 10^{9} / 1$ (IQR: $3.437-6.432 \times 10^{9} /$ ), the median lymphocyte count was $1.497 \times 10^{9} /$ I (IQR: $1.113-1.860 \times$ $\left.10^{9} / \mathrm{l}\right)$, the median monocyte count was $0.462 \times$ $10^{9} /$ (IQR: $0.224-0.660 \times 10^{9} /$ I) and the median NLR was 3.124 (IQR: 2.131-4.917).

We included all patients during a median follow-up period of 3.25 years (IQR: 3.0-4.1 years) $(n=306)$. The median time from first treatment to second treatment of retreatment patients was 1.25 years (IQR: $0.9-1.9$ years) $(n=68)$. The rest of the PTB cases who had completed anti-TB treatment and were cured without retreatment were selected as controls $(n=238)$. According to the receiver operating characteristics $(R O C)$ curve, the best cut-off value of NLR was 2.53 , as shown in Figure 2, with a sensitivity of $70.6 \%$ and a specificity of $45.4 \%$. Forty-eight (78\%) patients had an elevated pretreatment NLR (2.53) identified in retreatment cases compared to $130(54.6 \%)$ patients identified among controls $(p=0.017)$. The distribution of sex did not differ between cases and controls ( $p=0.834$ ) (Table II). Compared to controls, cases were more likely to be smokers ( $p=$ $0.012)$, aged $\geq 60(p=0.001)$ and with initial cavitation $(p<0.001)$. The prevalence of DM, cancer, $\mathrm{HBV} / \mathrm{HCV}$ and extra-pulmonary lesion were higher among cases than controls (13.2\% vs. 9.7\%, $8.8 \%$ vs. $3.4 \%, 17.6 \%$ vs. $10.1 \%, 23.5 \%$ vs. $19.3 \%$, respectively), but without significance.

NLR $\geq 2.53$ before anti-TB treatment was associated with retreatment of PTB both in the univari-

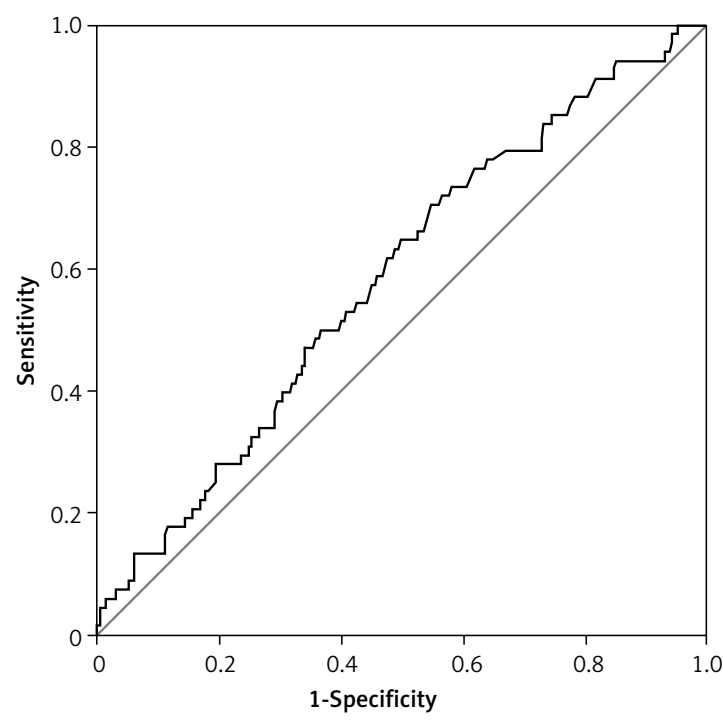

Figure 2. ROC curve of NLR between retreatment cases and no-retreatment controls

able analysis $(\mathrm{OR}=1.994,95 \% \mathrm{Cl}: 1.116-3.564)$ and the multivariable analysis (adjusted OR (AOR) $=2.409,95 \% \mathrm{Cl}: 1.212-4.788$ ) (Table III). In the multivariable analysis we included only 66 cases and 209 controls because of missing information in the bacteriological test and the results of chest X-ray. We conducted a separate analysis using all cases and controls and adjusting for all factors except for the bacteriological test and results of chest X-ray; the association was slightly attenuated but remained statistically significant (AOR = 2.157, 95\% Cl: 1.143-4.072). NLR $\geq 2.53$ before anti-TB treatment was a significant predictor for

Table II. Characteristics of study participants

\begin{tabular}{|c|c|c|c|}
\hline Parameter & $\begin{array}{c}\text { Cases }(N=68) \\
n(\%)\end{array}$ & $\begin{array}{c}\text { Controls }(N=238) \\
n(\%)\end{array}$ & $P$-value ${ }^{a}$ \\
\hline \multicolumn{4}{|l|}{ NLR: } \\
\hline$\geq 2.53$ & $48(78.0)$ & $130(54.6)$ & 0.017 \\
\hline$<2.53$ & $20(22.0)$ & $108(45.4)$ & \\
\hline \multicolumn{4}{|l|}{ Age [years]: } \\
\hline$<40$ & $7(10.3)$ & $60(25.2)$ & 0.001 \\
\hline $40-59$ & $15(22.1)$ & $75(31.5)$ & \\
\hline$\geq 60$ & $46(67.6)$ & $103(43.3)$ & \\
\hline Sex, male & $50(73.5)$ & $178(74.8)$ & 0.834 \\
\hline History of smoking & $42(61.2)$ & $106(44.5)$ & 0.012 \\
\hline Cancer & $6(8.8)$ & $8(3.4)$ & 0.078 \\
\hline $\mathrm{DM}$ & $9(13.2)$ & $23(9.7)$ & 0.408 \\
\hline $\mathrm{HBV} / \mathrm{HCV}$ & $12(17.6)$ & $25(10.1)$ & 0.125 \\
\hline Coexisting extra-pulmonary lesion & $16(23.5)$ & $46(19.3)$ & 0.453 \\
\hline Culture positive and/or smear-positive ${ }^{b}$ & $32(48.5)$ & $95(42.9)$ & 0.431 \\
\hline Cavitation on initial $\mathrm{CXR}^{\mathrm{c}}$ & $32(47.1)$ & $65(29.1)$ & $<0.001$ \\
\hline
\end{tabular}

${ }^{a}$ By Pearson's $\chi^{2}$ test, ${ }^{b}$ missing information in 2 cases and 17 controls, 'missing information in 15 controls. 
Table III. Univariable and multivariable odds ratios for the associations between potential risk factors and TB retreatment

\begin{tabular}{|c|c|c|c|c|}
\hline Parameter & $\begin{array}{l}\text { Unadjusted odds } \\
\text { ratio }(95 \% \mathrm{Cl})\end{array}$ & $P$-value & $\begin{array}{l}\text { Adjusted odds ratio } \\
\qquad(95 \% \mathrm{Cl})\end{array}$ & $P$-value \\
\hline \multicolumn{5}{|l|}{ NLR: } \\
\hline$<2.53$ & Reference & & Reference & \\
\hline$\geq 2.53$ & $1.994(1.116-3.564)$ & 0.020 & $2.409(1.212-4.788)$ & 0.012 \\
\hline \multicolumn{5}{|l|}{ Age [years]: } \\
\hline$<40$ & Reference & & Reference & \\
\hline $40-59$ & $1.714(0.657-4.474)$ & 0.271 & $1.678(0.578-4.870)$ & 0.341 \\
\hline$\geq 60$ & $3.828(1.626-9.015)$ & 0.002 & $2.931(1.122-7.653)$ & 0.028 \\
\hline Sex, male & $0.936(0.507-1.729)$ & 0.833 & $0.428(0.190-0.963)$ & 0.040 \\
\hline History of smoking & $2.202(1.158-3.493)$ & 0.013 & $2.321(1.135-4.745)$ & 0.021 \\
\hline Cancer & $2.782(0.931-8.317)$ & 0.067 & $3.797(1.101-13.100)$ & 0.035 \\
\hline DM & $1.426(0.626-3.246)$ & 0.398 & $2.519(0.981-6.466)$ & 0.055 \\
\hline $\mathrm{HBV} / \mathrm{HCV}$ & $1.826(0.864-3.860)$ & 0.115 & $4.551(1.791-11.563)$ & 0.001 \\
\hline Coexisting extra-pulmonary lesion & $1.284(0.673-2.451)$ & 0.448 & $2.304(1.073-4.974)$ & 0.032 \\
\hline Culture positive and/or smear-positive ${ }^{a}$ & $1.768(0.978-3.198)$ & 0.059 & $0.755(0.382-1.491)$ & 0.418 \\
\hline Initial cavitation ${ }^{b}$ & $2.922(1.654-5.411)$ & $<0.001$ & $2.482(1.230-5.007)$ & 0.011 \\
\hline
\end{tabular}

a Missing information in 19 patients ( 2 cases and 17 controls), ${ }^{b}$ missing information in 15 patients (all controls).

PTB retreatment. Additionally, cavitation on chest $\mathrm{X}$-ray $(\mathrm{OR}=2.922,95 \% \mathrm{Cl}: 1.654-5.411 ; \mathrm{AOR}=$ $2.482,95 \% \mathrm{Cl}: 1.230-5.007)$, history of smoking $(\mathrm{OR}=2.202,95 \% \mathrm{Cl}: 1.158-3.493 ; \mathrm{AOR}=2.321$, $95 \% \mathrm{Cl}: 1.135-4.745)$ and age $\geq 60(\mathrm{OR}=3.828$, $95 \% \mathrm{Cl}: 1.626-9.015 ; \mathrm{AOR}=2.931,95 \% \mathrm{Cl}: 1.122-$ 7.653) were also risk factors for PTB retreatment. Patients with cancer $(\mathrm{AOR}=3.797,95 \% \mathrm{Cl}: 1.101-$ 13.10), $\mathrm{HBV} / \mathrm{HCV}(\mathrm{AOR}=4.551,95 \% \mathrm{Cl}: 1.791-$ 11.56) and coexistence of extra-pulmonary lesions $(A O R=2.304,95 \% \mathrm{Cl}: 1.073-4.974)$ were associated with PTB retreatment in the multivariable analyses but not in the univariable analysis. However, gender, bacteriological confirmation and DM were not confirmed to be factors associated with PTB retreatment in this study.

When we used a different definition for PTB retreatment, the increased risk of NLR $\geq 2.53$ on TB retreatment was consistently observed. The OR was largest when the date of the second treatment was defined as more than 3 months after the first treatment $(A O R=2.409,95 \% \mathrm{Cl}: 1.212$ 4.788, Table IV). The OR was almost the same when we defined the date of the second treatment as more than 6 months after the first treatment ( $A O R=2.256,95 \% \mathrm{Cl}: 1.128-4.512)$. Finally, the OR was decreased but not much when we prolonged the duration between the second and first treatment to more than 1 year (AOR $=2.191,95 \%$ $\mathrm{Cl}: 1.027-4.677)$. Above all, the result of increased risk of NLR $\geq 2.53$ on TB retreatment was stable.

The retreatment rate during the follow-up among all cases is shown in Figure $3 \mathrm{~A}$. The retreatment rates were $0 \%$ after 3 months, $2.9 \%$ after 6 months, 3.9\% after 1 year and $6.9 \%$ after 2 years. The retreatment rate increased with the follow-up time. The retreatment rates in PTB patients with NLR $\geq 2.53$ and NLR $<2.53$ are shown in Figure $3 \mathrm{~B}$; they were $27.1 \%$ and $15.5 \%$, respectively, with a significant difference (log-rank test; $p=0.010$ ).

\section{Discussion}

The PTB retreatment still remains a major health problem despite an increase in coverage of

Table IV. Association of NLR with TB retreatment by different definitions for TB retreatment

\begin{tabular}{|lccc|}
\hline Parameter & $\begin{array}{c}\text { No. of cases } \\
\text { with NLR } \geq 2.53 \\
n(\%)\end{array}$ & $\begin{array}{c}\text { No. of controls with } \\
\text { NLR } \geq 2.53 \\
n(\%)\end{array}$ & $\begin{array}{c}\text { Adjusted odds ratio } \\
(95 \% \mathrm{Cl})\end{array}$ \\
\hline Three months after first treatment & $48(72.7)$ & $114(54.5)$ & $2.409(1.212-4.788)$ \\
\hline Six months after first treatment & $44(71.0)$ & $114(54.5)$ & $2.256(1.128-4.512)$ \\
\hline One year after first treatment & $35(71.4)$ & $114(54.5)$ & $2.191(1.027-4.677)$ \\
\hline
\end{tabular}


A

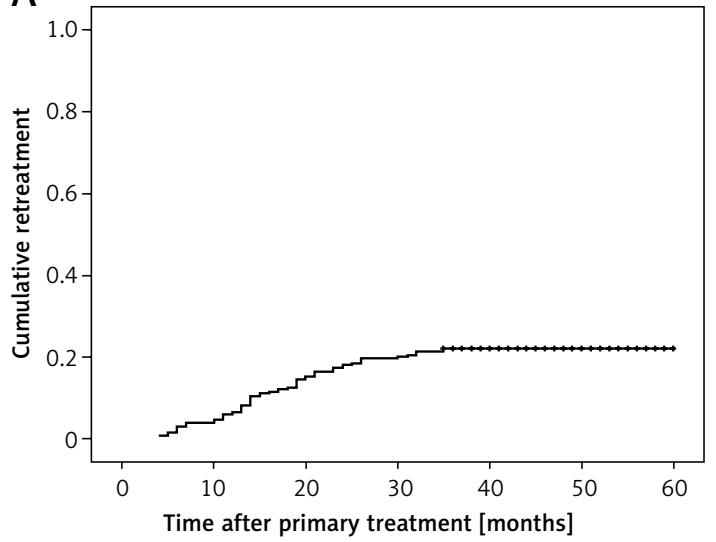

B

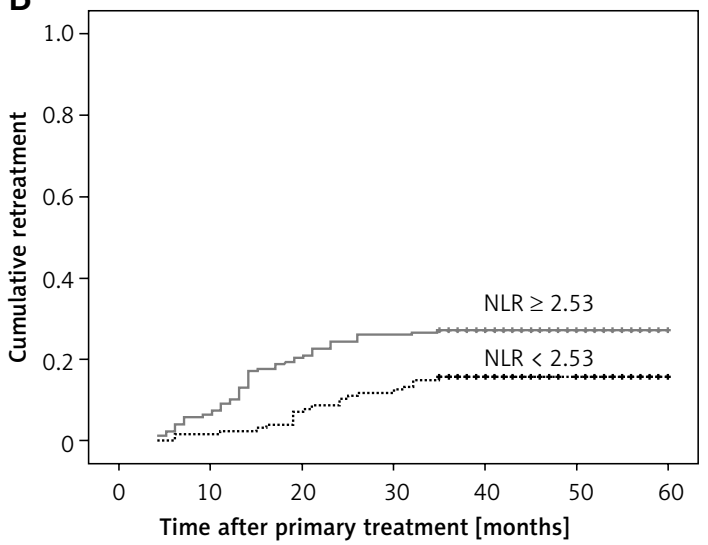

Figure 3. Cumulative retreatment with months after primary treatment. A - Cumulative retreatment during follow-up period in 306 PTB patients (Kaplan-Meier); B - Cumulative retreatment during follow-up period in PTB patients with NLR $\geq 2.53$ and NLR $<2.53$ (Kaplan-Meier) (log-rank test; $p=0.010$ )

DOTS therapy. Better understanding of the determinants of TB retreatment helps to identify those at high risk of retreatment disease and promises to reduce the disease burden through risk factor intervention.

White blood cell populations play an important role in the systemic inflammatory response to infection $[16,17]$. Following infection, the number of circulating neutrophils increases while lymphocyte counts decrease [16]. Neutrophilia is well recognized as an infection marker, whereas absolute lymphocytopenia is ignored as a possible marker in infectious disease management. Recently, the latter showed its potential value in predicting bacteremia and the severity of several infectious diseases [18-22].

Combining both parameters as the ratio of neutrophil and lymphocyte counts is increasingly used in several clinical circumstances. Primarily, NLR was studied as an infection marker and found to correlate well with disease severity and outcome $[19,23,24]$. For example, NLR was reported to be significantly higher in patients with advanced PTB as opposed to patients with mild to moderate PTB [25]. Moreover, NLR may be treated as a possible marker in differentiating tuberculosis from bacterial community-acquired pneumonia (CAP) [26] and sarcoidosis [27]. Recently, NLR was suggested as a simple marker that may discriminate severe bacterial from viral infections [28]. Other studies focused on the use of the NLR in specific clinical conditions, or its use as an independent predictor of survival in patients with various conditions ranging from oncological to cardiovascular diseases [29-32].

The enhanced neutrophil response might promote Mycobacterium tuberculosis (Mtb) proliferation and transmission, and the suppression of lymphocytes might inhibit the anti-Mtb immune response and delay therapy duration, which to- gether lead to a high NLR may be a novel index to evaluate PTB retreatment. In this case-control study, we determined NLR $\geq 2.53$ as a risk factor for PTB retreatment, and, when we used a different definition for PTB retreatment, the result of increased risk of NLR $\geq 2.53$ on PTB retreatment was stable. To our knowledge, we are the first to propose that NLR $\geq 2.53$ is predictive of PTB retreatment. Moreover, a history of smoking, initial cavitation on chest $X$-ray and age $\geq 60$ were also confirmed to be factors associated with PTB retreatment.

Our study also has limitations. The eligible patients represented a fraction of the patients diagnosed with active tuberculosis during the study period, raising a concern for a selection bias. As a retrospective study, collecting information from medical records filled in by other professionals is unavoidable but tends to be less accurate. Fortunately, at the time of clinical assessment, although clinicians were not blinded to the full blood count, they were not aware of this hypothesis, thus diminishing diagnostic bias. Other potential risk factors associated with TB in mainly new patients have been described in other settings, such as health knowledge, distance to treatment center, and patients' economic status; these could not be evaluated in this study [33, 34].

In conclusion, the result of increased risk of NLR $\geq 2.53$ on PTB retreatment was stable. NLR $\geq 2.53$ is predictive of PTB retreatment. Additionally, the retreatment rate in PTB patients with NLR $\geq 2.53$ was higher than in PTB patients with NLR $<2.53$. Other factors, especially initial cavitation on chest X-ray, history of smoking, and age $\geq 60$, also had higher risk for retreatment. Due to the large number of PTB patients and the limited cure rate for PTB retreatment in China, the high cost of retreatment should not be ignored. We recommend that, upon completion of anti-TB treatment, 
follow-up strategies targeting PTB patients with $N L R \geq 2.53$ must pay more attention to avoid TB relapse, treatment default and abandonment related to retreatment early. Moreover, further studies of chest X-ray monitoring are needed in PTB patients with initial cavitation to control the risk of retreatment. Adequate blood test and chest X-ray monitoring in PTB patients in this cohort study were highlighted to avoid PTB retreatment, issues requiring urgent attention.

\section{Acknowledgments}

Yongmei Yin, Shougang Kuai and Jun Liu contributed equally to this article.

We thank all the patients for their support in this research and colleagues of The Fifth People's Hospital of Wuxi for their assistance in collection of research data. This work was supported by the Science and Technology Bureau of Wuxi, China (No. CSEOIN1226) and the Health Bureau of Wuxi, China (No. Q201506 and No. Z201405).

\section{Conflict of interest}

The authors declare conflict of interest.

\section{References}

1. World Health Organization. Global tuberculosis report 2014. Geneva: WHO.

2. Wang L, Zhang H, Ruan Y, et al. Tuberculosis prevalence in China, 1990-2010: a longitudinal analysis of national survey data. Lancet 2014; 383: 2057-64.

3. World Health Organisation. Global Tuberculosis report 2012. Geneva WHO

4. Crofts JP, Andrews NJ, Barker RD, Delpech V, Abubakar I. Risk factors for recurrent tuberculosis in England and Wales, 1998-2005. Thorax 2010; 65: 310-14.

5. World Health Organization. Multidrug and extensively drug-resistant TB (M/XDR-TB): 2010 global report on surveillance and response. Geneva WHO.

6. Murray J, Sonnenberg P, Shearer SC, Godfrey-Faussett P. Human immunodeficiency virus and the outcome of treatment for new and recurrent pulmonary tuberculosis in African patients. Am J Respir Crit Care Med 1999; 159: 733-40.

7. Tozkoparan E, Deniz O, Ucar E, Bilgic H, Ekiz K. Changes in platelet count and indices in pulmonary tuberculosis. Clin Chem Laboratory Med 2007; 45: 1009-13.

8. Unsal E, Aksaray S, Köksal D, Sipit T. Potential role of interleukin 6 in reactive thrombocytosis and acute phase response in pulmonary tuberculosis. Postgrad Med J 2005; 81: 604-7.

9. Sahin F, Yazar E, Yildiz P. Prominent features of platelet count, plateletcrit, mean platelet volume and platelet distribution width in pulmonary tuberculosis. Multidisciplinary Respir Med 2012; 7: 38.

10. Sahin F, Yıldız P. Distinctive biochemical changes in pulmonary tuberculosis and pneumonia. Arch Med Sci 2013; 9: 656-61.

11. Toh E, Wilson J, Sebag-Montefiore D, Botterill I. Neutrophil-lymphocyte ratio (NLR) as a simple and novel biomarker for prediction of locoregional recurrence after chemoradiotherapy for squamous cell carcinoma (SCC) of the anus. Colorectal Dis 2014; 16: 90-7.

12. Szkandera J, Absenger G, Liegl-Atzwanger B, et al. Elevated preoperative neutrophil/lymphocyte ratio is associated with poor prognosis in soft-tissue sarcoma patients. Br J Cancer 2013; 108: 1677-83.

13. Lee S, Oh SY, Kim SH, et al. Prognostic significance of neutrophil lymphocyte ratio and platelet lymphocyte ratio in advanced gastric cancer patients treated with FOLFOX chemotherapy. BMC Cancer 2013; 13: 350.

14. Chua W, Charles KA, Baracos VE, Clarke SJ. Neutrophil/ lymphocyte ratio predicts chemotherapy outcomes in patients with advanced colorectal cancer. Br J Cancer 2011; 104: 1288-95.

15. Stotz M, Gerger A, Eisner F, et al. Increased neutrophillymphocyte ratio is a poor prognostic factor in patients with primary operable and inoperable pancreatic cancer. Br J Cancer 2013; 109: 416-21.

16. Jilma B, Blann A, Pernerstorfer T, et al. Regulation of adhesion molecules during human endotoxemia. No acute effects of aspirin. Am J Respir Crit Care Med 1999; 159: 857-63.

17. Kiran B, Cagatay T, Clark P, et al. Can immune parameters be used as predictors to distinguish between pulmonary multidrug-resistant and drug-sensitive tuberculosis? Arch Med Sci 2010; 6: 77-82.

18. Wyllie DH, Bowler IC, Peto TE. Bacteraemia prediction in emergency medical admissions: role of $C$ reactive protein. J Clin Pathol 2005; 58: 352-6.

19. Zahorec R. Ratio of neutrophil to lymphocyte counts-rapid and simple parameter of systemic inflammatiion and stress in critically ill. Bratisl Lek Listy 2001; 102: 5-14.

20. Wyllie DH, Bowler IC, Peto TE. Relation between lymphopenia and bacteraemia in UK adults with medical emergencies. J Clin Pathol 2004; 57: 950-5.

21. Hawkins CA, Collignon P, Adams DN, Bowden FJ, Cook MC. Profound lymphopenia and bacteraemia. Intern Med J 2006; 36: 385-8.

22. de Jager CP, van Wijk PT, Mathoera RB, et al. Lymphocytopenia and neutrophil-lymphocyte count ratio predict bacteremia better than conventional infection markers in an emergency care unit. Crit Care 2010; 14: R192.

23. Knaus WA, Draper EA, Wagner DP, Zimmerman JE. APACHE II: a severity of disease classification system. Crit Care Med 1985; 13: 818-29.

24. Vincent JL, Moreno R, Takala J, et al. The SOFA (Sepsis-related Organ Failure Assessment) score to describe organ dysfunction/failure. On behalf of the working group on sepsis-related problems of the European Society of Intensive Care Medicine. Intensive Care Med 1996; 22: 707-10.

25. Abakay O, Abakay A, Sen HS, Tanrikulu AC. The relationship between inflammatory marker levels and pulmonary tuberculosis severity. Inflammation 2015; 38: 691-6.

26. Yoon NB, Son C, Um SJ. Role of the neutrophil-lymphocyte count ratio in the differential diagnosis between pulmonary tuberculous is and bacterial community-acquired pneumonia. Ann Labor Med 2013; 33: 105-10.

27. Iliaz S, Iliaz R, Ortakoylu G, et al. Value of neutrophil/ lymphocyte ratio in the differential diagnosis of sarcoidosis and tuberculosis. Ann Thorac Med 2014; 9: 232-5.

28. Holub M, Beran O, Kaspříková N, Chalupa P. Neutrophil to lymphocyte count ratio as a biomarker of bacterial infections. Cent Eur J Med 2012; 7: 258-61.

29. Gibson PH, Croal BL, Cuthbertson BH, et al. Preoperative neutrophil-lymphocyte ratio and outcome from coronary artery bypass grafting. Am Heart J 2007; 154: 995-1002. 
30. Tamhane UU, Aneja S, Montgomery D, et al. Association between admission neutrophil to lymphocyte ratio and outcomes in patients with acute coronary syndrome. Am J Cardiol 2008; 102: 653-7.

31. Halazun KJ, Hardy MA, Rana AA, et al. Negative impact of neutrophil-lymphocyte ratio on outcome after liver transplantation for hepatocellular carcinoma. Ann Surg 2009; 250: 141-51.

32. Sarraf KM, Belcher E, Raevsky E, et al. Neutrophil/lymphocyte ratio and its association with survival after complete resection in non-small cell lung cancer. J Thorac Cardiovasc Surg 2009; 137: 425-8.

33. Bam TS, Gunneberg C, Chamroonsawasdi K, et al. Factors affecting patient adherence to DOTS in urban Kathmandu. Nepal Int I Tuberc Lung Dis 2006; 10: 270-6.

34. Chang KC, Leung CC, Tam CM. Risk factors for defaulting from anti-tuberculosis treatment under directly observed treatment in Hong Kong. Int J Tuberc Lung Dis 2004; 8: 1492-8. 